\title{
Effects of Channel Estimation Errors on V-BLAST Detection
}

\author{
Wei Peng ${ }^{1}$, Fumiyuki Adachi ${ }^{1}$, Shaodan $\mathrm{Ma}^{2}$, Jiangzhou Wang ${ }^{3}$ and Tung-Sang $\mathrm{Ng}^{2}$ \\ ${ }^{1}$ Department of Electrical and Communication Engineering, Tohoku University, Japan \\ ${ }^{2}$ Department of Electrical and Electronic Engineering, the University of Hong Kong, Hong Kong \\ ${ }^{3}$ Department of Electronics, the University of Kent, UK
}

\begin{abstract}
This paper investigates the effects of channel estimation errors on zero-forcing (ZF) vertical Bell laboratories layered space time (V-BLAST) detection. An analytical method is presented to derive the symbol error probability (SEP) of the signals detected at each stage. The effects of imperfect channel estimation on the SEP performance of V-BLAST detection are then studied. It is shown that ZF-VBLAST detection is very sensitive to the channel estimation errors under high signal to noise ratio (SNR). It is also shown that when optimal ordering is adopted, the effects of channel estimation errors are more significant on the latter detection stages.
\end{abstract}

Index Terms - Imperfect Channel Estimation, MIMO Multiplexing, Symbol Error Probability, V-BLAST detection.

\section{INTRODUCTION}

The vertical Bell laboratories layered space time (VBLAST) algorithm is a popular detection algorithm for multiple input multiple output (MIMO) multiplexing systems, since it is capable of achieving a substantial part of MIMO capacity with a low implementation complexity [1-2]. Due to the non-linear nature of interference cancellation, its performance analysis has been considered difficult and most of the existing analytical works make the assumption of perfect channel state information at the receiver (CSIR) [3-6]. However, channel estimation errors usually exist. Their presence in general would affect the system performance. It is thus important to theoretically investigate the effects of channel estimation errors on the performance of V-BLAST detection.

In this paper, the zero forcing (ZF) V-BLAST detection with imperfect channel estimation is considered. An analytical method is presented to derive the symbol error probability (SEP) of the signals detected at each stage, by which the effects of channel estimation errors are studied.

The rest of the paper is organized as follows. Section II describes the MIMO multiplexing system model and the ZF V-BLAST detection algorithm. The SEP performance of ZFVBLAST detection is analyzed in Section III. Then, the effects of channel estimation errors are investigated in Section IV. Finally, conclusions are drawn in Section V.

\section{SyStem MODEL AND ZF-VBLAST DETECTION}

\section{A. System model}

Consider a MIMO multiplexing system with $N_{t}$ transmit and $N_{r}$ receive antennas $\left(N_{t} \leq N_{r}\right)$. The base-band received signal vector is given by

$$
\mathbf{y}=\mathbf{H x}+\mathbf{n},
$$

where $\mathbf{y}=\left[y_{1}, \cdots, y_{N_{r}}\right]^{T}$ is an $N_{r} \times 1$ vector with $y_{j}$ being the received signal at the $j^{\text {th }}$ receive antenna; superscript $T$ represents matrix transpose; $\mathbf{n}=\left[n_{1}, \cdots, n_{N_{r}}\right]^{T}$ is an $N_{r} \times 1$ complex additive white Gaussian noise (AWGN) vector, each element being independent with zero mean and variance $\sigma_{n}^{2}$; $\mathbf{H}$ is an $N_{r} \times N_{t}$ channel matrix whose $(j, i)^{t h}$ element $h_{j, i}$ stands for the channel gain from the $i^{t h}$ transmit antenna to the $j^{\text {th }}$ receive antenna and is assumed to be an independently and identically distributed (i.i.d) complex Gaussian variable with zero mean and unit variance $\left(\sigma_{h}^{2}=1\right) ; \mathbf{x}=\left[x_{1}, \cdots, x_{N_{t}}\right]^{T}$ is an $N_{t} \times 1$ vector with the $i^{\text {th }}$ element being the transmitted signal from the $i^{\text {th }}$ transmit antenna and independent from the other elements. Let $C$ represent the constellation of the transmitted signals. It is assumed that all the constellation symbols have equal probability. For simple analysis, the transmitted signals are assumed to be quadrature phase shift keying (QPSK) modulated. Note that the analysis can be easily extended to the system using other modulation schemes.

\section{B. Channel estimation errors}

Let $\hat{\mathbf{H}}$ be the estimated channel matrix. It is generally assumed that the elements of $\hat{\mathbf{H}}$ are independent complex Gaussian variables with zero mean and variance $\sigma_{\hat{h}}^{2}$. It follows from [7] that the channel matrix $\mathbf{H}$ can be written as

$$
\mathbf{H}=\rho_{h, \hat{h}} \hat{\mathbf{H}}+\Delta \mathbf{H}
$$

where $\rho_{h, \hat{h}}=c_{h, \hat{h}} \sigma_{h} / \sigma_{\hat{h}}, c_{h, \hat{h}}$ is the correlation coefficient between the corresponding elements of $\hat{\mathbf{H}}$ and $\mathbf{H}$,

$$
c_{h, \hat{h}}=\frac{1}{N_{r} N_{t}} \cdot \sum_{j=1}^{N_{r}} \sum_{i=1}^{N_{t}} \frac{E\left\{h_{j, i} \hat{h}_{j, i}^{\dagger}\right\}}{\sqrt{E\left\{\left|h_{j, i}\right|^{2}\right\}} \cdot \sqrt{E\left\{\left|\hat{h}_{j, i}^{\dagger}\right|^{2}\right\}}} .
$$

where superscript $†$ denotes conjugate; $E\{\cdot\}$ denotes expectation and $|\cdot|$ denotes absolute. $c_{h, \hat{h}}$ is a positive real and $0<c_{h, \hat{h}} \leq 1 . \Delta \mathbf{H}$ represents the channel estimation error matrix whose $(j, i)^{t h}$ element equals to $\Delta h_{j, i}=h_{j, i}-\rho_{h, h} \hat{h}_{j, i}$. Previous study in [7] has shown that the channel estimation error $\Delta h_{j, i}$ can be modeled as an i.i.d complex Gaussian variable with zero mean and its variance equals $\sigma_{\Delta h}^{2}=\operatorname{var}\left(h_{j, i}-\rho_{h, \hat{h}} \hat{h}_{j, i}\right)=\sigma_{h}^{2}\left(1-c_{h, \hat{h}}^{2}\right)$. When perfect channel estimation is achieved at the receiver, $\rho_{h, \hat{h}}=c_{h, \hat{h}}=1$. 
It should be noted that $\rho_{h, \hat{h}}$ and $c_{h, \hat{h}}$ are determined by the channel estimator. For any given SNR and channel estimator, $\rho_{h \hat{h}}$ and $c_{h, \hat{h}}$ are assumed to be known at the receiver [8].

Substituting (2) into (1), it yields

$$
\mathbf{y}=\left(\rho_{h, \hat{h}} \hat{\mathbf{H}}+\Delta \mathbf{H}\right) \mathbf{x}+\mathbf{n}=\rho_{h, \hat{h}} \hat{\mathbf{H}} \mathbf{x}+\mathbf{u},
$$

where $\mathbf{u}=\Delta \mathbf{H x}+\mathbf{n}$ stands for the channel-estimation-errorinduced interference plus noise and can be regarded as the equivalent noise vector with the $j^{\text {th }}$ element as $u_{j}=\sum_{i=1}^{N_{t}} \Delta h_{j, i} x_{i}+n_{j}$. Since $\Delta h_{j, i}$ and $n_{j}$ are independent zero mean complex Gaussian variables with variance $\sigma_{\Delta h}^{2}$ and $\sigma_{n}^{2}$ respectively, it follows that for given $\mathbf{x}, u_{j}$ is also Gaussian variable [9] with zero mean and the variance of $u_{j}$ is given by

$$
\begin{aligned}
\sigma_{u}^{2} & =\operatorname{var}\left(\sum_{i=1}^{N_{t}} \Delta h_{j, i} x_{i}+n_{j}\right) \\
& =\sum_{i=1}^{N_{t}}\left|x_{i}\right|^{2} \sigma_{\Delta h}^{2}+\sigma_{n}^{2} \\
& =E_{s}\left(1-c_{h, \hat{h}}^{2}\right)+\sigma_{n}^{2}
\end{aligned}
$$

where $E_{s}=\sum_{i=1}^{N_{t}}\left|x_{i}\right|^{2}$ denotes the total transmit power at the transmitter.

\section{ZF-VBLAST Detection}

After ordering, the received signal vector in (4) can be rewritten as

$$
\mathbf{y}=\rho_{h, h} \hat{\mathbf{H}} \mathbf{P} \mathbf{P}^{T} \mathbf{x}+\mathbf{u}=\rho_{h, h} \overline{\mathbf{H}} \overline{\mathbf{x}}+\mathbf{u},
$$

where $\mathbf{P}$ is a permutation matrix. $\overline{\mathbf{H}}=\hat{\mathbf{H}} \mathbf{P}$ represents the ordered estimated channel matrix and $\overline{\mathbf{x}}=\mathbf{P}^{T} \mathbf{x}=\left[\bar{x}_{1}, \cdots, \bar{x}_{N_{t}}\right]^{T}$ is the corresponding ordered transmitted signal vector.

To proceed, the ordered estimated channel matrix $\overline{\mathbf{H}}$ is $\mathrm{QR}$ decomposed as $\overline{\mathbf{H}}=\overline{\mathbf{Q}} \overline{\mathbf{R}}$ where $\overline{\mathbf{Q}}$ is an $N_{r} \times N_{t}$ matrix with $\overline{\mathbf{Q}}^{H} \overline{\mathbf{Q}}=\mathbf{I}$ [10]; superscript $H$ represents conjugate transpose; $\overline{\mathbf{R}}$ is an $N_{t} \times N_{t}$ upper triangular matrix. Then, a vector $\mathbf{z}$ is formed by left multiplying the received signal vector with $\overline{\mathbf{Q}}^{H}$, given by

$$
\mathbf{z}=\overline{\mathbf{Q}}^{H}\left(\rho_{h, h} \overline{\mathbf{H}} \overline{\mathbf{x}}+\mathbf{u}\right)=\rho_{h, h} \overline{\mathbf{R}} \overline{\mathbf{x}}+\mathbf{g},
$$

where $\mathbf{g}=\overline{\mathbf{Q}}^{H} \mathbf{u}$. In fact, the elements of $\mathbf{g}$ are still complex Gaussian variables with zero mean and variance $\sigma_{g}^{2}=\sigma_{u}^{2}$ since the statistic properties of $\mathbf{u}$ is maintained by unitary transform [11].

Given (7), the ZF V-BLAST detection can be carried out sequentially in $N_{t}$ stages as follows. At the $1^{\text {st }}$ stage, the signal is detected as

$$
\tilde{\bar{x}}_{N_{t}}=\operatorname{slice}\left(\hat{\bar{x}}_{N_{t}}\right)
$$

where $\tilde{\bar{x}}_{N_{t}}$ stands for the decision of $\bar{x}_{N_{t}} ; \hat{\bar{x}}_{N_{t}}=z_{N_{t}} / \rho_{h, \hat{h}} \bar{r}_{N_{t}, N_{t}}$ is the signal estimate calculated from (7), $z_{j}$ is the $j^{\text {th }}$ element of $\mathbf{z}$ and $\bar{r}_{j, i}$ is the $(j, i)^{\text {th }}$ element of $\overline{\mathbf{R}}$; Symbol slice $(\cdot)$ represents the operation to map the symbol in the bracket to its nearest point in the constellation. At the $i^{\text {th }}$ stage ( $i \geq 2$ ), the interference from the previous $i-1$ ordered transmitters is subtracted based on the decisions $\left(\tilde{\bar{x}}_{N_{t}}, \tilde{\bar{x}}_{N_{t}-1}, \cdots, \tilde{\bar{x}}_{N_{t}-i+2}\right)$ made in the previous stages and the signal $\bar{x}_{N_{t}-i+1}$ is then detected according to (7) as

$$
\tilde{\bar{x}}_{N_{t}-i+1}=\operatorname{slice}\left(\hat{\bar{x}}_{N_{t}-i+1}\right) \text {, }
$$

$\begin{aligned} & \text { where } \\ & \hat{\bar{x}}_{N_{t}-i+1}\end{aligned}=\left(z_{N_{t}-i+1}-\sum_{j=N_{t}-i+2}^{N_{t}} \rho_{h, \hat{h}} \bar{r}_{N_{t}-i+1, j} \tilde{\bar{x}}_{j}\right) / \rho_{h, \hat{h}} \bar{r}_{N_{t}-i+1, N_{t}-i+1}$.

\section{SEP DERIVATION}

Considering the $i^{\text {th }}$ detection stage, it is clear that $\bar{x}_{N_{t}-i+1}$ is the transmitted signal to be detected and $X_{N_{t}-i+2, \cdots, N_{t}}=\left\{\tilde{\bar{x}}_{N_{t}-i+2}, \cdots, \tilde{\bar{x}}_{N_{t}}\right\}$ includes the decisions made in the previous $i-1$ stages. The SEP of $\bar{x}_{N_{t}-i+1}$ can be written in terms of the SEPs conditioned on the decision errors corresponding to $X_{N_{t}-i+2, \cdots, N_{t}}$ as [12]

$$
\begin{aligned}
& P\left(\tilde{\bar{x}}_{N_{t}-i+1} \neq \bar{x}_{N_{t}-i+1}\right) \\
= & P\left(\tilde{\bar{x}}_{N_{t}-i+1} \neq \bar{x}_{N_{t}-i+1} \mid \operatorname{err}_{i-1}(\varnothing)\right) P\left(\operatorname{err}_{i-1}(\varnothing)\right) \\
& +\sum_{k=N_{t}-i+2}^{N_{t}} P\left(\tilde{\bar{x}}_{N_{t}-i+1} \neq \bar{x}_{N_{t}-i+1} \mid \operatorname{err}_{i-1}(k)\right) P\left(\operatorname{err}_{i-1}(k)\right) \\
& +\sum_{l=N_{t}-i+2,}^{N_{t}} \sum_{m=l+1}^{N_{t}} P\left(\tilde{\bar{x}}_{N_{t}-i+1} \neq \bar{x}_{N_{t}-i+1} \mid \operatorname{err}_{i-1}(l, m)\right) P\left(\operatorname{err}_{i-1}(l, m)\right) \\
& +\cdots+P\left(\tilde{\bar{x}}_{N_{t}-i+1} \neq \bar{x}_{N_{t}-i+1} \mid \operatorname{err}_{i-1}(\Omega)\right) P\left(\operatorname{err}_{i-1}(\Omega)\right)
\end{aligned}
$$

In (10), $\operatorname{err}_{i-1}(\cdot)$ generally stands for an error event of $X_{N_{\omega}-i+2, \cdots, N_{t}}$ which happens in the previous $(i-1)^{t h}$ stages; $P\left(\bar{x}_{N_{t}-i+1} \neq \bar{x}_{N_{t}-i+1} \mid \operatorname{err}_{i-1}(\cdot)\right)$ and $P\left(\operatorname{err}_{i-1}(\cdot)\right)$ represent the SEP conditioned on $\operatorname{err}_{i-1}(\cdot)$ and the probability of $\operatorname{err}_{i-1}(\cdot)$, respectively. In more detail, $\operatorname{err}_{i-1}(\varnothing), \operatorname{err}_{i-1}(k), \operatorname{err}_{i-1}(l, m)$ and $\operatorname{err}_{i-1}(\Omega)$ respectively represent no error, one error $\left(\tilde{\bar{x}}_{k} \neq \bar{x}_{k}\right)$, two errors $\left(\tilde{\bar{x}}_{l} \neq \bar{x}_{l}, \tilde{\bar{x}}_{m} \neq \bar{x}_{m}\right)$ and all errors in $X_{N_{t}-i+2, \cdots, N_{t}}$.

For simplicity, the ZF V-BLAST detection without ordering is considered first, i.e., $\mathbf{P}=\mathbf{I}$. In this situation, $\overline{\mathbf{x}}=\mathbf{P}^{T} \mathbf{x}=\left[x_{1}, \cdots, x_{N_{t}}\right]^{T}$. Namely, the detection order is from $x_{N_{t}}$ to $x_{1}$. The extension to the ZF V-BLAST detection with optimal ordering will be considered at the end of this section.

\section{A. The conditional error probability}

It has been found in [13] that the probability distribution function (p.d.f) of the normalized square of the diagonal element $\omega_{i}\left(\omega_{i}=\left|r_{i, i}\right|^{2} / \sigma_{\hat{h}}^{2}\right)$ is

$$
p\left(\omega_{i}\right)=\frac{\omega_{i}^{N_{r}-i} \exp \left(-\omega_{i}\right)}{\left(N_{r}-i\right) !}, i=1,2, \cdots, N
$$

\section{1) SEP conditioned on err $_{i-1}(\varnothing)$}

The SEP conditioned on $\operatorname{err}_{i-1}(\varnothing)$ is derived first and the result in this sub-section will form the basis for the following derivation on the SEPs conditioned on the other error events.

Recall that at the $i^{\text {th }}$ detection stage, the decision of $\bar{x}_{N_{t}-i+1}$

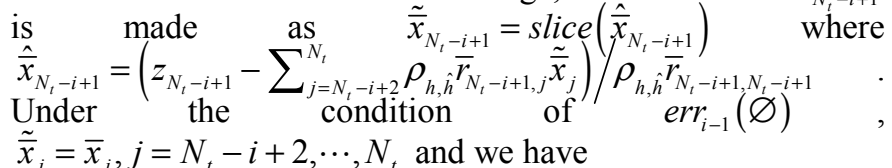




$$
\tilde{\bar{x}}_{N_{t}-i+1}=\operatorname{slice}\left(\frac{\rho_{h, \hat{h}} \overline{\hat{h}}_{N_{t}-i+1, N_{t}-i+1} \bar{x}_{N_{t}-i+1}+g_{N_{t}-i+1}}{\rho_{h, h} \bar{h}_{N_{t}-i+1, N_{t}-i+1}}\right) \text {. }
$$

Given (12), the post-detection SNR can be calculated by

$$
\begin{aligned}
& \gamma_{N_{t}-i+1, e r_{i_{-1}}(\varnothing)} \\
& =\frac{\rho_{h, \hat{h}}^{2} \bar{r}_{N_{t}-i+1, N_{t}-i+1}^{2}\left|\bar{x}_{N_{t}-i+1}\right|^{2}}{\sigma_{g}^{2}}=\frac{c_{h, \hat{h}}^{2} \omega_{N_{t}-i+1}\left|\bar{x}_{N_{t}-i+1}\right|^{2}}{\sigma_{u}^{2}} .
\end{aligned}
$$

It follows that the SEP conditioned on $\bar{x}_{N_{t}-i+1}, \omega_{N_{t}-i+1}$ and $\operatorname{err}_{i-1}(\varnothing)$ is given as [10]

$$
\begin{aligned}
& p\left(\tilde{\bar{x}}_{N_{t}-i+1} \neq \bar{x}_{N_{t}-i+1} \mid \bar{x}_{N_{t}-i+1}, \omega_{N_{t}-i+1}, \operatorname{err}_{i-1}(\varnothing)\right) \\
& =G\left(\gamma_{N_{t}-i+1, e r r_{i-1}(\varnothing)}\right) \\
& =2 Q\left(\sqrt{\gamma_{N_{t}-i+1, e r r_{i-1}(\varnothing)}}\right)-Q^{2}\left(\sqrt{\gamma_{N_{t}-i+1, e r r_{i-1}(\varnothing)}}\right)
\end{aligned}
$$

where $Q(A)=1 / \sqrt{2 \pi} \cdot \int_{A}^{\infty} \exp \left(-t^{2} / 2\right) d t$. Note that the function depends on the modulation scheme. It is straight forward to apply this method to the systems using other modulation schemes by altering $G(\cdot)$. Since $Q(A)$ can be rewritten as $Q(A)=1 / \pi \cdot \int_{0}^{\pi / 2} \exp \left(-A^{2} / 2 \sin ^{2} \theta\right) d \theta$ [14], the conditional SEP in (14) is equivalent to

$$
\begin{aligned}
& p\left(\tilde{\bar{x}}_{N_{t}-i+1} \neq \bar{x}_{N_{t}-i+1} \mid \bar{x}_{N_{t}-i+1}, \omega_{N_{t}-i+1}, \operatorname{err}_{i-1}(\varnothing)\right) \\
= & \frac{1}{\pi} \int_{0}^{3 \pi / 4} \exp \left(-\frac{\gamma_{N_{t}-i+1, e r_{i-1}}(\varnothing)}{2 \sin ^{2} \theta}\right) d \theta \\
= & \frac{1}{\pi} \int_{0}^{3 \pi / 4} \exp \left(-\frac{c_{h, \hat{h}}^{2} \omega_{N_{t}-i+1}\left|\bar{x}_{N_{t}-i+1}\right|^{2}}{2 \sigma_{u}^{2} \sin ^{2} \theta}\right) d \theta
\end{aligned}
$$

By averaging (15) with respect to the statistics of $\bar{x}_{N_{t}-i+1}$ and $\omega_{N_{t}-i+1}$, the average conditional SEP can be obtained as

$$
\begin{aligned}
& P\left(\tilde{\bar{x}}_{N_{t}-i+1} \neq \bar{x}_{N_{t}-i+1} \mid \operatorname{err}_{i-1}(\varnothing)\right)= \\
& \sum_{x_{N_{t}-i t+1} \in}\left[p\left(\bar{x}_{N_{t}-i+1}\right) \cdot \int_{0}^{\infty} p\left(\tilde{\bar{x}}_{N_{t}-i+1} \neq \bar{x}_{N_{t}-i+1} \mid \bar{x}_{N_{t}-i+1}, \omega_{N_{t}-i+1}, \operatorname{err}_{i-1}(\varnothing)\right),\right. \\
& \left.\cdot p\left(\omega_{N_{t}-i+1}\right) d \omega_{N_{t}-i+1}\right]
\end{aligned}
$$

where $p\left(\bar{x}_{N_{t}-i+1}\right)$ and $p\left(\omega_{N_{t}-i+1}\right)$ are the p.d.f of $\bar{x}_{N_{t}-i+1}$ and $\omega_{N_{t}-i+1}$, respectively. Substituting (11) into (16), the average conditional SEP becomes

$$
\begin{aligned}
& P\left(\tilde{\bar{x}}_{N_{t}-i+1} \neq \bar{x}_{N_{t}-i+1} \mid \operatorname{err}_{i-1}(\varnothing)\right) \\
& =\frac{1}{\pi} \sum_{x_{N_{t}-i+1} \in C} p\left(\bar{x}_{N_{t}-i+1}\right) \cdot \int_{0}^{3 \pi / 4}\left(1+\frac{c_{h, \hat{h}}^{2}\left|\bar{x}_{N_{t}-i+1}\right|^{2}}{2 \sigma_{u}^{2} \sin ^{2} \theta}\right)^{-\left(N_{r}-N_{t}+i\right)} d \theta .
\end{aligned}
$$

Note that $\int_{0}^{\infty} x^{p} \exp (-\mu x) d x=p ! \mu^{-n-1}$ [15] is used in the above derivation.

\section{2) SEPs conditioned on the other error events}

Under the condition of $\operatorname{err}_{i-1}(k), \quad \tilde{\bar{x}}_{k} \neq \bar{x}_{k} \quad$ and $\tilde{\bar{x}}_{q}=\bar{x}_{q}\left(q=N_{t}-i+2, \cdots, N_{t}, q \neq k\right)$. It follows from (7) and (9) that

$$
\begin{aligned}
\tilde{\bar{x}}_{N_{t}-i+1} & =\text { slice }\left(\frac{\rho_{h, \hat{h}} \bar{r}_{N_{t}-i+1, N_{t}-i+1} \bar{x}_{N_{t}-i+1}+\rho_{h, \hat{h}} \bar{r}_{N_{t}-i+1, k} \Delta \bar{x}_{k}+g_{N_{t}-i+1}}{\rho_{h, \hat{h}} \bar{r}_{N_{t}-i+1, N_{t}-i+1}}\right), \\
= & \text { slice }\left(\frac{\rho_{h, \hat{h}} \bar{r}_{N_{t}-i+1, N_{t}-i+1} \bar{x}_{N_{t}-i+1}+v_{N_{t}-i+1, k}}{\rho_{h, \hat{h}} \bar{r}_{N_{t}-i+1, N_{t}-i+1}}\right)
\end{aligned}
$$

where $\Delta \bar{x}_{k}=\bar{x}_{k}-\tilde{\bar{x}}_{k}$ represents the decision error and $v_{N_{t}-i+1, k}=\rho_{h, h} \bar{r}_{N_{t}-i+1, k} \Delta \bar{x}_{k}+g_{N_{t}-i+1}$ includes the interference introduced by both error propagation and channel estimation errors. It follows that for a given $\Delta \bar{x}_{k}, v_{N_{t}-i+1, k}$ is also a complex Gaussian variable with zero mean and variance:

$$
\begin{aligned}
\sigma_{v_{N_{t}-i+1, k}}^{2} & =\operatorname{var}\left\{c_{h, \hat{h}} \bar{r}_{N_{t-i+1}, k} \Delta \bar{x}_{k} / \sigma_{\hat{h}}+g_{N_{t}-i+1}\right\} . \\
& =c_{h, \hat{h}}^{2}\left|\Delta \bar{x}_{k}\right|^{2}+\sigma_{g}^{2}=c_{h, \hat{h}}^{2}\left|\Delta \bar{x}_{k}\right|^{2}+\sigma_{u}^{2}
\end{aligned} .
$$

Since the symbol error with the highest probability occurs in adjacent positions in the constellation, it is assumed that error only happens between the transmitted symbol and its nearest constellation neighbor. Therefore, $\left|\Delta \bar{x}_{k}\right|^{2} \approx \min \left(d_{k, c}{ }^{2}\right)=\alpha_{k} E\left\{\left|\bar{x}_{k}\right|^{2}\right\}$, where $d_{k, c}^{2}$ represents the minimum squared Euclidean distance (SED) between $\bar{x}_{k}$ and its constellation neighbors; $E\left\{\left|\bar{x}_{k}\right|^{2}\right\}$ stands for the average symbol energy, and $\alpha_{k}$ varies with the modulation scheme. For QPSK modulation, $\min \left\{d_{k, c}^{2}\right\}=2 E\left\{\left|\bar{x}_{k}\right|^{2}\right\}$ and $\alpha_{k}=2$.

The average SEP conditioned on $\operatorname{err}_{i-1}(k)$ can be obtained similarly as the average SEP conditioned on $\operatorname{err}_{i-1}(\varnothing)$ in (13) by replacing $\sigma_{u}^{2}$ with $\sigma_{v_{N_{1}-i+1.1}}^{2}$. And the average conditional SEP $P\left(\tilde{\bar{x}}_{N_{t}-i+1} \neq \bar{x}_{N_{t}-i+1} \mid \operatorname{err} r_{i-1}(k)\right)$ is calculated from (17) by replacing $\sigma_{u}^{2}$ with $\sigma_{v_{N-1-i t, k}}^{2}$.

In the same way, the average SEPs under conditions of the other error events are derived as

$$
\begin{aligned}
& P\left(\tilde{\bar{x}}_{N_{t}-i+1} \neq \bar{x}_{N_{t}-i+1} \mid \operatorname{err}_{i-1}(l, m)\right) \\
= & \frac{1}{\pi} \sum_{x_{N_{t}-t+1} \in C} p\left(\bar{x}_{N_{t}-i+1}\right) \cdot \int_{0}^{3 \pi / 4}\left(1+\frac{c_{h, \hat{h}}^{2}\left|\bar{x}_{N_{t}-i+1}\right|^{2}}{2 \sigma_{v_{N_{t}-i+1, l, m}}^{2} \sin ^{2} \theta}\right)^{-\left(N_{r}-N_{t}+i\right)} d \theta \\
& P\left(\tilde{\bar{x}}_{N_{t}-i+1} \neq \bar{x}_{N_{t}-i+1} \mid \operatorname{err}_{i-1}(\Omega)\right) \\
= & \frac{1}{\pi} \sum_{x_{N_{t}-t+1} \in C} p\left(\bar{x}_{N_{t}-i+1}\right) \cdot \int_{0}^{3 \pi / 4}\left(1+\frac{c_{h, \hat{h}}^{2}\left|\bar{N}_{N_{t}-i+1}\right|^{2}}{2 \sigma_{v_{N_{t}-i t 1, \Omega}}^{2} \sin ^{2} \theta}\right)^{-\left(N_{r}-N_{t}+i\right)} d \theta
\end{aligned}
$$

where $\quad \sigma_{v_{N_{l}-i t 1, l, m}}^{2}=c_{h, \hat{h}}^{2}\left(\alpha_{l} E\left\{\left|\bar{x}_{l}\right|^{2}\right\}+\alpha_{m} E\left\{\left|\bar{x}_{m}\right|^{2}\right\}\right)+\sigma_{u}^{2} \quad$ and $\sigma_{v_{N_{t}-i+1, \Omega}}^{2}=c_{h, \hat{h}}^{2} \sum_{k=N_{t}-i+2,}^{N_{t}} \alpha_{k} E\left\{\left|\bar{x}_{k}\right|^{2}\right\}+\sigma_{u}^{2}$.

\section{B. The probabilities of the error events}

The error event $\operatorname{err}_{i-1}(\varnothing)$ is the joint event of $\tilde{\bar{x}}_{N_{t}-i+2}$ (the decision made at the $(i-1)^{\text {th }}$ stage) and $X_{N_{t}-i+3, \cdots, N_{t}}$ which happens in the previous $(i-2)^{\text {th }}$ stages. Consequently, the probability $P\left(\operatorname{err}_{i-1}(\cdot)\right)$ can be calculated sequentially in terms of $P\left(\operatorname{err}_{i-2}(\cdot)\right)$. For example, the probability of the error event $P\left(\operatorname{err}_{i-1}(\varnothing)\right)$ is given as: 


$$
\begin{aligned}
& P\left(\operatorname{err}_{i-1}(\varnothing)\right) \\
= & P\left(\tilde{\bar{x}}_{N_{t}-i+2}=\bar{x}_{N_{t}-i+2} \mid \operatorname{err}_{i-2}(\varnothing)\right) \cdot P\left(\operatorname{err}_{i-2}(\varnothing)\right) \\
= & {\left[1-P\left(\tilde{\bar{x}}_{N_{t}-i+2} \neq \bar{x}_{N_{t}-i+2} \mid \operatorname{err}_{i-2}(\varnothing)\right)\right] \cdot P\left(\operatorname{err}_{i-2}(\varnothing)\right) }
\end{aligned} .
$$

The probabilities of the other error events are obtained similarly. The average SEP $P\left(\tilde{\bar{x}}_{N_{t}-i+1} \neq \bar{x}_{N_{t}-i+1}\right)$ now can be obtained by substituting the conditional SEPs and the probabilities of the error events into (10).

\section{SEP derivation for the ZF V-BLAST detection with optimal ordering}

It was shown in [2] that the optimal detection order is chosen to maximize the post-detection SNR at each stage. Namely, the transmitted signals are optimally ordered according to their energy at the receiver in descending order (in this paper, it is assumed that ordering is perfect). When adopting this ordering rule, the detection order is accordingly determined by the norm of the column vectors of the channel matrix given that equal transmitting power is allocated to the transmit antennas.

This optimal ordering would change the statistics of the elements in the upper triangular matrix $\overline{\mathbf{R}}$ and thus affect the SEP performance at each stage. Taking a $2 \times N_{r}$ system as an example, the ordering mainly changes the statistics of $\bar{r}_{1,1}$ and $\bar{r}_{2,2}$. It is found from [4] that the p.d.f. of $\omega_{1}=\bar{r}_{1,1}^{2} / \sigma_{\hat{h}}^{2}$ and $\omega_{2}=\bar{r}_{2,2}^{2} / \sigma_{\hat{h}}^{2}$ after the optimal ordering would respectively change to

$$
p\left(\omega_{1}\right)=\frac{2 \omega_{1}^{N_{r}-1} \exp \left(-2 \omega_{1}\right)}{\left(N_{r}-1\right) !}\left(\sum_{m=0}^{N_{r}-1} \frac{1}{m !} \omega_{1}^{m}\right)
$$

and

$$
\begin{aligned}
& p\left(\omega_{2}\right)= \\
& \frac{2 \omega_{2}^{N_{r}-2}}{\left(N_{r}-2\right) !}\left[\begin{array}{l}
\exp \left(-\omega_{2}\right)- \\
\exp \left(-2 \omega_{2}\right) \sum_{m=0}^{N_{r}-1} \frac{1}{2^{k+1}} \sum_{k=0}^{k} \frac{1}{k !}\left(2 \omega_{2}\right)^{k}
\end{array}\right] .
\end{aligned}
$$

Using (22) and (23) instead of (11), the SEPs at the $1^{\text {st }}$ and $2^{\text {nd }}$ stages are obtained following the steps of the proposed method.

Remarks: The proposed method is applicable to the ZF VBLAST detection in a general system as long as the statistics of the elements in the upper triangular matrix are available. Unfortunately to the best knowledge of the authors, when optimal ordering is adopted, the statistics for systems with more than two inputs have yet to be found.

\section{EFFECTS OF CHANNEL ESTIMATION ERRORS}

The effects of channels estimation errors will be evaluated by numerical results calculated from (10). The parameters used to get the numerical results are set as follows. First VBLAST detection without ordering is considered. Two systems with $N_{t}=2, N_{r}=4$ and $N_{t}=4, N_{r}=4$ are taken as examples. Next the V-BLAST detection with optimal ordering is considered and a system with $N_{t}=2, N_{r}=4$ is taken as an example. The channel gain between each pair of transmit and receive antennas is a randomly generated complex Gaussian variable with zero mean and unit variance. Unit transmitting power is allocated to each antenna and QPSK modulation is used for each antenna. SNR is defined as the ratio of the average transmit power to the average noise power. The normalized channel estimation error $\varepsilon\left(\varepsilon=\sqrt{\sigma_{\Delta h}^{2} / \sigma_{h}^{2}}\right)$ varies from 0 (perfect channel estimation) to 0.3 . Since $\sigma_{\Delta h}=\sigma_{h} \sqrt{1-c_{h, \hat{h}}^{2}}=\varepsilon \sigma_{h}$, the parameter $c_{h, \hat{h}}$ can be calculated correspondingly. Note that small $\varepsilon$ corresponds to highaccuracy channel estimation and vice versa.

Fig. 1 and Fig. 2 show the SEP performance as a function of $\varepsilon$ for the systems with $N_{t}=2, N_{r}=4$ (without ordering, $\mathrm{SNR}=6 \mathrm{~dB}$ and $18 \mathrm{~dB}$ ) and $N_{t}=4, N_{r}=4$ (without ordering, $\mathrm{SNR}=10 \mathrm{~dB}$ and $30 \mathrm{~dB}$ ), respectively. It can be seen that: 1) the SEP performance at each stage degrades when the normalized channel estimation error $\varepsilon$ increases; 2) as SNR increases, the performance degradation due to imperfect channel estimation becomes more significant.

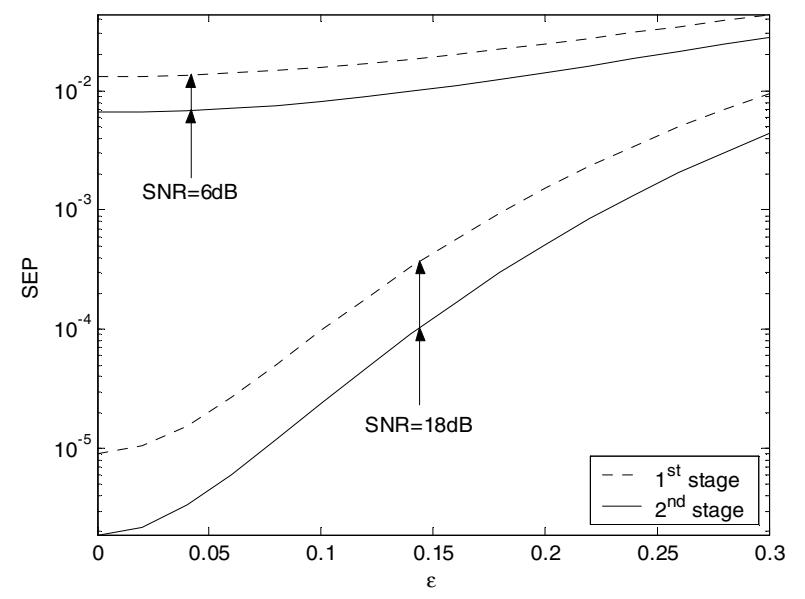

Fig. 1 Effect of channel estimation errors on the SEPs of the system with $N_{t}=2, N_{r}=4$, without ordering.

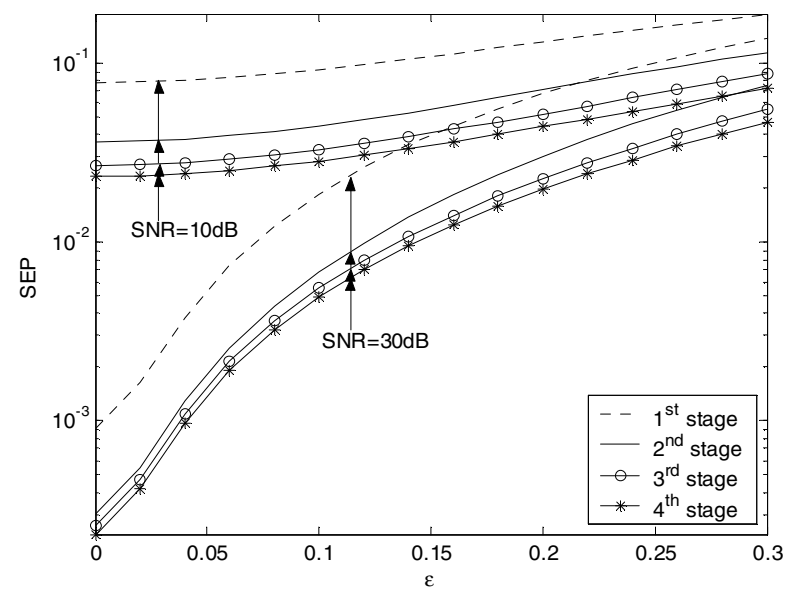

Fig. 2 Effect of channel estimation errors on the SEPs of the system with

$$
N_{t}=4, N_{r}=4 \text {, without ordering. }
$$

Fig. 3 shows the SEP performance as a function of $\varepsilon$ for the system $N_{t}=2, N_{r}=4$ (with optimal ordering, $\mathrm{SNR}=6 \mathrm{~dB}$ and $18 \mathrm{~dB}$ ). From Fig. 3, it is further observed that under 
higher SNR, when optimal ordering is adopted, the effect of channel estimation errors on the $2^{\text {nd }}$ stage is more significant than that on the $1^{\text {st }}$ detection stage. This is different from the detection without ordering. The reason is that under high SNR, when channel estimation error occurs, the $2^{\text {nd }}$ stage detection suffers from not only the channel estimation error directly but also the error propagation from the $1^{\text {st }}$ stage which is intensified by channel estimation errors. From this example, it is thus inferred that under higher SNR, when optimal ordering is adopted, the effects of channel estimation errors would be more significant on the latter detection stages.

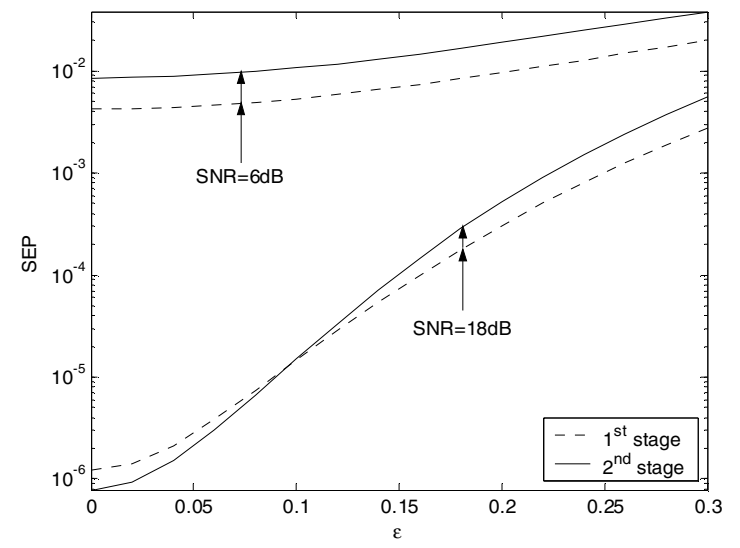

Fig. 3 Effect of channel estimation errors on the SEPs of the system with

$$
N_{t}=2, N_{r}=4 \text {, with ordering. }
$$

Such knowledge about the effects of channel estimation errors can be very useful to system designers. For example, the SEP performance as a function of SNR $\left(N_{t}=2, N_{r}=4\right.$, without ordering) is shown in Fig. 4. Suppose it is desired to have a $\mathrm{SEP}=10^{-3}$. From Fig. 4, the normalized channel estimation error $\varepsilon$ should be limited to 0.1 when $\mathrm{SNR}=10 \mathrm{~dB}$. On the other hand, $\varepsilon$ should be limited to 0.2 when $\mathrm{SNR}=14 \mathrm{~dB}$. Given these information, the system designer can then design an optimal power allocation scheme so that the total power of pilot and data signals will be minimized to achieve the required performance.

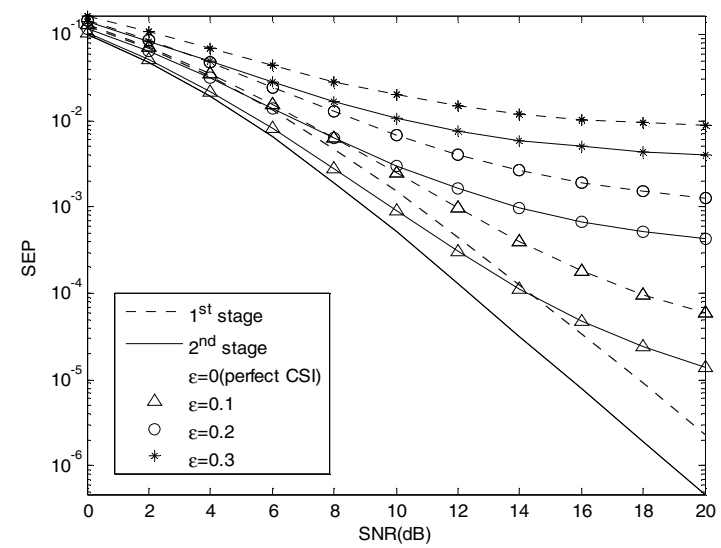

Fig. 4 SEP performance as a function of SNR, $N_{t}=2, N_{r}=4$, without ordering.

\section{CONCLUSIONS}

The effects of channel estimation errors on ZF-V-BLAST detection have been studied in the paper. An analytical method to derive the SEP of ZF V-BLAST detection has been presented and the effects of channel estimation errors have been investigated according to the analytical results. It has been shown that the performance of ZF-BLAST detection is more sensitive to the channel estimation errors when the signal to noise ratio (SNR) is higher. It has also been found that when optimal ordering is adopted, the effects of channel estimation errors are more significant on the latter detection stages.

\section{REFERENCES}

[1] G. J. Foschini, G. D. Golden, R. A. Valenzuela and P. W. Wolniansky, "Simplified Processing for High Spectral Efficiency Wireless Communication Employing Multi-Element Arrays," IEEE Journal on Selected Areas in Communications, vol. 17, pp. 1841-1852, November 1999.

[2] G. D. Golden, G. J. Foschini, R. A. Valenzuela, and P. W. Wolniansky, "Detection Algorithm and Initial Laboratory Results Using V-BLAST Space-Time Communication Architecture," IEEE Electronics Letters, vol. 35, pp. 14-16, November 1998.

[3] S. Loyka and F. Gagnon, "Performance Analysis of the V-BLAST Algorithm: An Analytical Approach," IEEE Transactions on Wireless Communications, vol. 3, pp. 1326-1337, July 2004.

[4] R. T. Xu and F. C. M. Au, "Analytical Approach of V-BLAST Performance with Two Transmit Antennas," IEEE Conference on Wireless Communications and Networking, vol. 1, pp. 396 - 401, March 2005.

[5] S. Loyka and F. Gagnon, "V-BLAST without Optimal ordering: Analytical Performance Evaluation for Rayleigh Fading Channels," IEEE Transactions on Wireless Communications, vol. 54, pp. 11091120, June 2006.

[6] C. Shen, Y. Zhu, S. D. Zhou and J. J. Jiang, "On the Performance of VBLAST with Zero-Forcing Successive Interference Cancellation Receiver," IEEE Globecom 2004, vol. 5, pp. 2818 - 2822, November 2004.

[7] W. Y. Li, N. C. Beaulieu, "Effects of Channel-Estimation Errors on Receiver Selection-Combining Schemes for Alamouti MIMO Systems with BPSK," IEEE Transactions on Communications, vol. 54, pp. 169178, January 2006.

[8] S. J. Grant and J. K. Cavers, "Performance Enhancement through Joint Detection of co-Channel Signals Using Diversity Arrays," IEEE Transactions on Communications, vol. 46, pp. 1038-1049, August 1998.

[9] J. G. Proakis, "Digital Communications," McGraw Hill, 4th edition, 2001.

[10] Winter and J. David, "Matrix Algebra," New York: Macmillan, 1992.

[11] V. V. Prasolov, "Problems and Theorems in Linear Algebra," American Mathematical Society, 1994.

[12] Henry, S. John and W. Woods, "Probability and Random Process with Applications to Signal Processing," Prentice Hall, 2002.

[13] T. W. Anderson, "An Introduction to Multivariate Analysis," 3rd edition, New York: Wiley, 2003.

[14] M. K. Simon and M. S. Alouini, "Digital Communication over Fading Channels," New York: Wiley, 2000.

[15] I. N. Bronshtein, K. A. Semendyayev, G. Musiol and H. Muehlig, "Handbook of Mathematics," Springer, 1997. 